\title{
Prospects for the measurement of the $b$-quark mass at the ILC
}

\author{
Seidai Tairafune, ${ }^{a, *}$ Juan Fuster, ${ }^{b}$ Adrian Irles, ${ }^{b}$ Germán Rodrigo, ${ }^{b}$ Marcel Vos, ${ }^{b}$ \\ Hitoshi Yamamoto $^{a b}$ and Ryo Yonamine ${ }^{a}$ \\ ${ }^{a}$ Tohoku University, \\ 6-3, Aramaki Aza-Aoba, Aoba-ku, Sendai 980-8578, Japan \\ ${ }^{b}$ IFIC, Universitat de València and CSIC \\ c./ Catedrático Josè Beltràn 2, E-46980 Paterna, Spain \\ E-mail: seidai@epx.phys.tohoku.ac.jp, juan.fuster@ific.uv.es, \\ adrian.irles@ific.uv.es, german.rodrigo@csic.es, marcel.vos@ific.uv.es, \\ hitoshi.yamamoto@ific.uv.es, yonamine@epx.phys.tohoku.ac.jp
}

Quark masses are one of the important parameters of the Standard Model, and $b$-quark mass has been measured at various energy scales so far, and these results agree with QCD running mass prediction. This paper reports an assessment result of $b$-quark mass measurement in future highenergy electron-positron colliders through the ILD full detector simulation. We present prospects for the measurement of the ratio of the three-jet rates $R_{3}^{b l}$ as the observable to extract $b$-quark mass and estimated dominant uncertainties. It turns out that the precision of $b$-quark mass is $1 \mathrm{GeV}$ for the ILC $250 \mathrm{GeV}$ run with the luminosity of $2 \mathrm{ab}^{-1}$. Based on this result, we also estimated the $b$-quark mass precision for the Giga-Z option, and it is $0.12 \mathrm{GeV}$. Thus, it is $\sim 3$ times superior to the precision of LEP and SLD measurements.

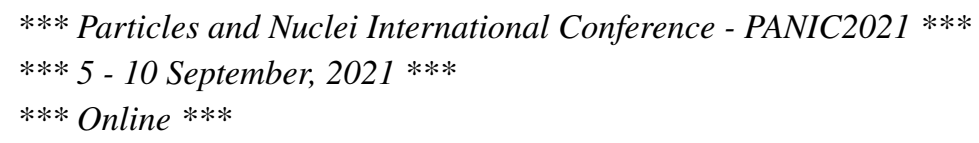

\footnotetext{
${ }^{1}$ On behalf of the ILD concept group

${ }^{2}$ We would like to thank the LCC generator working group and the ILD software working group for providing the simulation and the Monte Carlo samples in this study. We acknowledge the following financial supports: GP-PU(Graduate Program on Physics for the Universe) of Tohoku University (S. Tairafune), Projects FPA2015-65652-C4-3-R (MINECO/FEDER) and PGC2018-094856-B-100, PROMETEO-2018/060 (Generalitat Valenciana), and the iLINK grant (CSIC) (A. Irles and M.Vos), Generalitat Valenciana (Spain) under the grant number CIDEGENT/2020/21 (A. Irles), The Spanish Government (Agencia Estatal deInvestigación) and ERDF funds from European Commission (Grant No. FPA2017-84445-P), Generalitat Valenciana (Grant No. PROMETEO/2017/053), and the COST Action CA16201 PARTICLEFACE (G. Rodrigo).

${ }^{*}$ Speaker
} 


\section{Measurement of Running $b$-quark Mass with the Three-jet Ratio $R_{3}^{b l}$}

$\overline{\mathrm{MS}}$ running quark mass that is a quark mass definition has energy scale dependence. Therefore, precise measurements of $b$-quark mass at various higher energy scales verify QCD mass running prediction. For example, [1] proposed a method to extract $b$-quark mass at Z-pole energy scale by using double-ratio of exclusive hadronic observables:

$$
R_{3}^{b l}=\frac{R_{3}^{b}}{R_{3}^{l}}=\frac{\Gamma_{3 j}^{b}\left(y_{c}\right) / \Gamma^{b}}{\Gamma_{3 j}^{l}\left(y_{c}\right) / \Gamma^{l}},
$$

where $\Gamma_{3 j}^{q}\left(y_{c}\right)$ is the decay width of $Z \rightarrow q \bar{q} \rightarrow$ three jets for a quark flavor $q$ and $\Gamma^{q}$ is the total decay width of $Z \rightarrow q \bar{q}$. Here, flavor $l$ means light quarks $u, d$, s, and $y_{c}$ is a jet resolution parameter. Taking double-ratio has theoretical and experimental advantages: cancels electroweak, detector and hadronization corrections.

The DELPHI, ALEPH, OPAL and SLD collaborations performed $b$-quark mass measurements with Z-pole data. For example, DELPHI collaboration published the result [2] of

$$
m_{b}\left(M_{Z}\right)=2.85 \pm 0.18 \text { (stat.) } \pm 0.13 \text { (exp.) } \pm 0.19 \text { (had.) } \pm 0.12 \text { (theo.), }
$$

and these results agree with the QCD prediction.

Measurements of $b$-quark mass at higher energy scale above Z-pole at a new electron-positron collider are important works for further QCD mass running prediction and exploring the viability of new physics. We estimate systematic uncertainties in $b$-quark mass at $250 \mathrm{GeV} \mathrm{ILC} \mathrm{and} \mathrm{Giga-Z}$ ILC by ILD full detector simulation and assess the potential of a future electron-positron collider.

\section{The $b$-quark Mass Sensitivity on Three-jet Ratio $R_{3}^{b l}$}

In general, at higher center-of-mass energy experiments, since quark masses become relatively smaller compared with the center-of-mass energies, the mass effects should be smaller in observables. The $b$-quark mass sensitivity on $R_{3}^{b l}$ can be derived from the perturbative expression of $R_{3}^{b l}$ [3], and the fig.1 shows its theoretical predictions. These plots mean that if we want the precision of $0.4 \mathrm{GeV}$, we need to measure $R_{3}^{b l}$ with a precision of $1 \%$ for Z-pole and $0.1 \%$ for $250 \mathrm{GeV}$.

\section{Setup of $b$-quark Mass Measurement at $250 \mathrm{GeV}$}

The signal event in this analysis is $e^{+} e^{-} \rightarrow q \bar{q}(q=u, d, s, b)$, and there are several types of background events; radiative return: $e^{+} e^{-} \rightarrow q \bar{q} \gamma$ and the boson pair events: $e^{+} e^{-} \rightarrow W W / Z Z / Z H$ to four quarks.

This paper reports the results with the total luminosity of $2 \mathrm{ab}^{-1}$ with two polarization configurations of ILC beams: $\left(P_{e^{-}}, P_{e^{+}}\right)=(-0.8,+0.3)$ and $(+0.8,-0.3)$. We used the samples generated by the ILD concept group, and these events are simulated at LO for massless quarks with the WHIZARD 1.95 event generator. The parton showering and hadronization are simulated in the Pythia 6.422, and the mass effects are implemented in only the Pythia. The LO calculation lacks precision and can not provide the accurate prediction of $R_{3}^{b l}$, and we used the Monte Carlo simulation for assessment of the systematic errors on $b$-quark mass. The implementation of NLO calculations in the WHIZARD 2 is ongoing by the collaboration with the WHIZARD authors. 


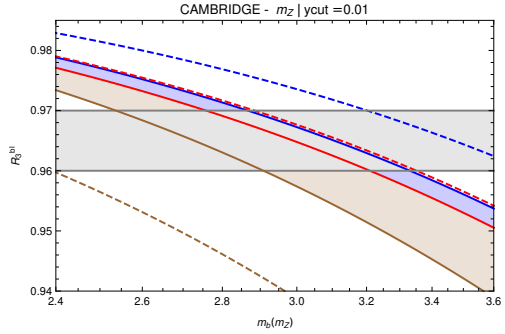

(a) $\sqrt{s}=M_{Z}$

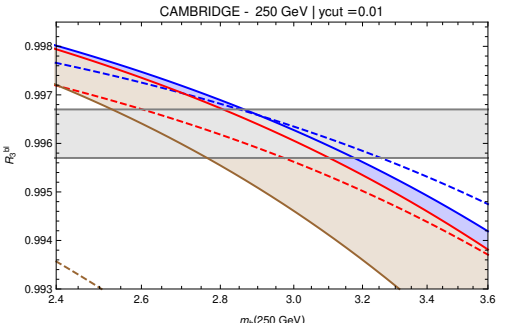

(b) $\sqrt{s}=250 \mathrm{GeV}$

Figure 1: Theoretical predictions of $R_{3}^{b l}$ as a function of running $b$-quark mass with Cambridge algorithm[3]. The dashed lines are LO, and the solid lines are NLO calculations. The brown lines are the predictions in terms of the pole mass and $\mu=\sqrt{s}$. The blue and red lines are the predictions in terms of the running mass and $\mu=2 \sqrt{s}$ and $\mu=\sqrt{s} / 2$, respectively. The horizontal gray band is an assumed experimental uncertainty.

\section{Event Selection}

Jet Reconstruction Reconstructed particles are forced to bundle into two jets according to the generalized $k_{\mathrm{T}}$ algorithm for $e^{-} e^{+}$colliders with optimizing parameters $R=1.25$ and $p=1$.

Flavor-Tagging Since $R_{3}^{b l}$ requires the identification of $b$-quark or $u d s$-quarks, we used the likelihoods of $b$-quark and $c$-quark, and we imposed $b$-likelihood $>0.85$ for the $b$-quark events, and $b$-likelihood $<0.4$ and $c$-likelihood $<0.25$ for the $u d s$-quark events.

Radiative return cuts Almost all ISR photons of radiative return events escape to beam pipe, but to reduce such invisible photons, we construct the ISR energy from two reconstructed jets kinematically and impose the condition that should be smaller than $50 \mathrm{GeV}$. Furthermore, additional cuts of neutral reconstructed particles are applied to reduce detected photons.

Boson pair events cut Boson pair productions can be cut by the form variable called thrust that means the sharpness of events, and applied the selection of Thrust $>0.85$.

\section{Assessment of Uncertainties and Prospects}

The observable $R_{3}^{b l}$ is measured after the above selection, and the left plot of the fig.2 shows the results for several different levels; parton, hadron, reconstructed levels. We estimated systematic uncertainties from correction factors $C^{\text {had }}$ and $C^{\text {det }}$ between different levels:

$$
\left.R_{3}^{b l}\right|_{\text {parton }}=C^{\text {had }} \times C^{\text {det }} \times\left. R_{3}^{b l}\right|_{\text {reco }} .
$$

Hadronization error We extrapolated half of LEP's result thanks to the progression of the study of hadronization and the higher energy collision than the LEP's time. Since the hadronization error at LEP is $0.2 \%$ on $C^{\text {had }}$, we assumed the uncertainty of $0.1 \%$ as hadronization effect.

Detector error We estimated the detector uncertainties by propagating the uncertainties on flavor-tagging efficiency $(0.1-0.5 \%)$ and background contaminations $(\mathrm{O}(0.1 \%))$ to the detector correction $C^{\text {det }}$ with Toy-MC.

Based on them, the $b$-quark mass precision at ILC $250 \mathrm{GeV}$ with the total statistics of $2 \mathrm{ab}^{-1}$ $\mathrm{H} 20$ scenario is obtained, and total error is $1 \mathrm{GeV}$ :

$$
\Delta m_{b}(250 \mathrm{GeV})=0.76(\text { stat. }) \pm 0.59(\text { exp. }) \pm 0.34 \text { (had.) } \pm 0.07 \text { (theo.) } \mathrm{GeV} .
$$



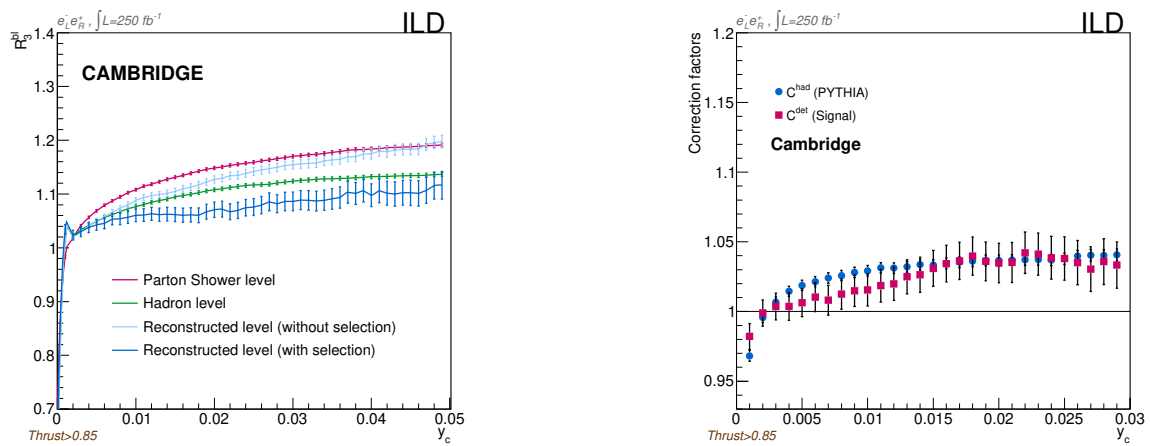

Figure 2: Left: $R_{3}^{b l}$ at parton(red), hadron(green) and reconstructed(blue) levels. Right: Correction factors $C^{\text {had }}$ and $C^{\text {det }}$ for only signal with $100 \%$ beam polarization.

As discussed in the section.2, this $250 \mathrm{GeV}$ measurement has the limited $b$-quark mass sensitivity, but this result extends the further QCD verification at never proved energies. On the other hand, if we assume the same detector performance as ILC $250 \mathrm{GeV}$ for Giga-Z run, it provides $\sim 3$ times better results thanks for 100 times more significant statistics:

$$
\Delta m_{b}\left(M_{Z}\right)=0.02 \text { (stat.) } \pm 0.02 \text { (exp.) } \pm 0.09 \text { (had.) } \pm 0.06 \text { (theo.) } \mathrm{GeV} \text {. }
$$

The fig. 3 summarizes the $b$-quark mass evolution that includes this work.

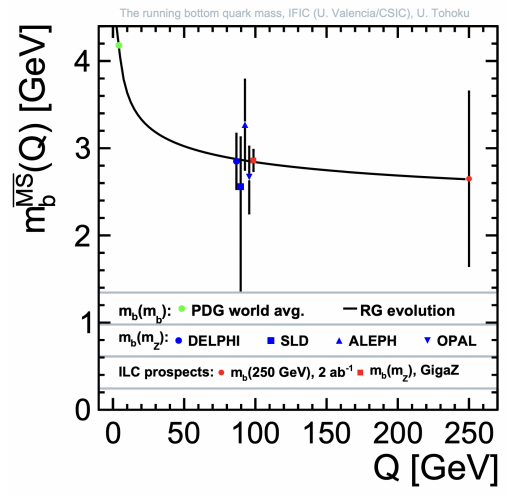

Figure 3: Red markers shows this work. Blue markers are LEP/SLC measurements at Z-pole, and the green marker is the PDG world average at the $m_{b}$ sclae.

\section{References}

[1] M. S. Bilenky, G. Rodrigo and A. Santamaria, Three jet production at LEP and the bottom quark mass, Nucl. Phys. B439 (1995) 505-535.

[2] DELPHI collaboration, J. Abdallah et al., Determination of the $b$ quark mass at the $M(Z)$ scale with the DELPHI detector at LEP, Eur. Phys. J. C46 (2006) 569-583, [hep-ex/0603046]

[3] J. Fuster, A. Irles, G. Rodrigo, S. Tairafune, M. Vos, H. Yamamoto, R. Yonamine, Prospects for the measurement of the b-quark mass at the ILC, arXiv:2104.09924 [hep-ex]. 